\title{
Immunohistochemical Localization of Aspergillus and p53 in Human Lung Tissues
}

\author{
Mohannad G. Al-Saghir ${ }^{1 *}$, Mosleh A. Alkhatatbeh ${ }^{2}$, Ahed J. Alkhatib ${ }^{3}$ \\ ${ }^{1}$ Department of Biological Sciences, Ohio University Zanesville, Zanesville, Ohio, USA \\ ${ }^{2}$ Department of Biological Sciences, Jordan University of Science and Technology, Irbid, Jordan \\ ${ }^{3}$ Department of Toxicology and Forensic Sciences, Jordan University of Science and Technology, Irbid, Jordan \\ Email: al-saghi@ohio.edu
}

Received 13 August 2015; accepted 6 October 2015; published 9 October 2015

Copyright (C) 2015 by authors and Scientific Research Publishing Inc.

This work is licensed under the Creative Commons Attribution International License (CC BY).

http://creativecommons.org/licenses/by/4.0/

(c) (i) Open Access

\section{Abstract}

Aspergilli are filamentous fungi which can cause opportunistic infections in Acquired Immunodeficiency Syndrome (AIDS) patients. Aspergilli can be found in human tissues either in the form of spores or hyphae. p53 is a tumor suppressor gene located in the short arm of chromosome 17. It is a potent transcriptional regulator of genes which are involved in many cellular activities including cell cycle arrest, apoptosis and angiogenesis. A loss of tumor suppressor function of p53 is the most common event leading to the development of human cancers. The rate by which p53 has a homology between different species has been reported from human to other vertebrates, it has been reported that it is available within Drosophila melanogaster and C. elegans [1] [2]. The aim of this study is to check if p53 is localized within Aspergilli or not using immunohistochemical techniques and study the relationship between Aspergilli infection and $\mathrm{p}^{53}$ in human lung tissues. 45 different samples of lung tissues, diagnosed as being none tumor, were taken randomly during the year of 2003-2004 from the autopsy cases submitted to the forensic medicine center in Irbid, Jordan. The sample group consisted of 12 females and 33 males. Labeled Streptavidin Biotin (LSAB) method and Mach-4 method were used to determine the Aspergilli infection and p53. The results show that the Aspergillus is presented in all used samples (100\% of the infection) in the form of spores or hyphae and all infected samples have mutant p53 molecules (p53 was located in Aspergillus spores and hyphae). According to this study, it is safe to posit that the mutant p53 molecules may be used by Aspergillus for its multiplication. Seemingly it is a biological behavior of Aspergillus to produce p53. The fate of the p53 is questioned, is it going to interact with the human cells initiating cancer? Further experimental investigations are required to determine such pathway. In conclusion, this study shows that Aspergillus is a producing agent for p53 and Aspergillus pathogenicity is caused by production of p53.

${ }^{*}$ Corresponding author.

How to cite this paper: Al-Saghir, M.G., Alkhatatbeh, M.A. and Alkhatib, A.J. (2015) Immunohistochemical Localization of Aspergillus and p53 in Human Lung Tissues. American Journal of Molecular Biology, 5, 117-123. 
Keywords

Aspergilius, p53, Immunohistochemical, Lung Tissues

\section{Introduction}

Aspergilli are filamentous fungi which can cause opportunistic infections in Acquired Immunodeficiency Syndrome (AIDS) patients [3]. Systemic fungal infections are also associated with the long term use of steroid, broad spectrum antibiotics, immunosuppressive drugs and cytotoxic agents [4]. Aspergilli can be found in human tissues either in the form of spores or hyphae [4].

The genus Aspergillus includes over 185 species, about 20 of them are reported to cause infections in human population. Among these, Aspergillus fumigatus is the most commonly isolated species, followed by Aspergillus flavus and Asperillus niger. Aspergillus spores (2 to $3 \mu \mathrm{m}$ ) are widely spread in the environment including the air therefore enter human lungs or through wounds [5].

From a pathological point of view, Aspergilli start as spores, which in turn, produce toxins giving the chance for hyphea to predominate. Aspergilli exert their pathogenesis via the production of mycotoxins. Among these mycotoxins, the well known aflatoxin is produced mostly by Aspergillus flavus. Mycotoxins have proven to possess carcinogenic potential [6]. By which, Aspergilli exert their pathogenesis that at long term process may lead to mutations in p53 and lung tumor is a result [7]. Diagnosis is made by screening of serum Aspergillus antibody, or culture of sputum. In case of lung tissue, diagnosis is achieved by demonstration of the Aspergilli in histological slides by Giemsa stain or silver methenamine stain. These techniques are not highly specific. In this study, Aspergillus is localized by specific immunohistochemistry using lung tissue.

p53 is a tumor suppressor gene located in the short arm of chromosome 17. It is a potent transcriptional regulator of genes which are involved in many cellular activities including cell cycle arrest, apoptosis and angiogenesis [8]. A loss of tumor suppressor function of p53 is the most common event leading to the development of human cancers [9]. Genetic alterations in p53 have been identified in $>50 \%$ of different types of human cancer including cancer of the lung. Mutations of the p53 gene are one of the most common genetic abnormalities found in all types of tumors of the lung, suggesting a crucial role in bronchial carcinogenesis. The extent of p53 mutations, however, varies among different types of cancer of the lung (70\% in cases of small cell undifferentiated carcinoma compared to $47 \%$ in cases of non-small cell lung cancer, including $65 \%$ of cases of squamous cell carcinoma [SCC] of the lung and $60 \%$ of cases of large cell carcinoma, but only $33 \%$ of cases of adenocarcinomas) [10].

The rate by which p53 has a homology between different species has been reported from human to other vertebrates, it has been reported that it is available within Drosophila melanogaster and C. elegans [1] [2].

The aim of this study is to check if p53 is localized within Aspergilli or not using immunohistochemical techniques and study the relationship between Aspergilli infection and p53 in human lung tissues.

\section{Materials and Methods}

\subsection{Tissue Samples and Patient Population}

45 different lung tissues, diagnosed as being none tumor, were taken randomly during the year of 2003-2004 from the autopsy cases submitted to the forensic medicine center in Irbid, Jordan. The sample group consisted of 12 females and 33 males. Information concerning diagnosis date, age, sex, and nature of specimens were obtained from patient files.

\subsection{Antibodies: Immunohistochemical Detection of Mutated p53}

Aspergillus antigen was performed using commercial available mouse monoclonal antibodies specific to mutated p53 (provided by santacruise biotechnology) was diluted 1:500 using revival series as the diluent, Anti-Aspergillus antibody (DAKO corporation, Carrpinteria, CA, USA) with dilution of 1:50.

The p53 antibody reacts with the wild type and mutant type of the p53 protein, whereas the wild type is not detectable because of its short half-life and low content. So in this work the detection was for mutant p53. 


\subsection{Immunohistochemical Staining}

Immunohistochemical staining is a simple and useful method for evaluating the expression of markers at the cellular level. Immunohistochemical detection of aspergillus and mutated p53 was demonstrated by using the universal labeled streptavidin biotin reagents (LSAB-Method) and compared to Mach-4 detection system (provided by Biocare Medical), which is composed of two parts: a probe which detects the primary antibody and horseradish peroxidase. Each solution was applied for 10 minutes followed by washing with PBS, followed by DAKO DAB Chromogen (DAKO Corporation, Carrpinteria, CA, USA). Immunostaining was followed by counter staining with hematoxylin (30 seconds). All slides were examined by Light Compound Microscopy.

\subsection{Labeled Streptavidin Biotin (LSAB) Method}

Sections $(4-\mu \mathrm{m})$ of routine pathological, formalin-fixed, paraffin-embedded lung tissues were mounted on glass slides coated with vecta bond. The sections were deparaffinized within the oven at $60^{\circ} \mathrm{C}$ for 30 minutes. Then the same sections were hydrated through xyline to water. The Sections were subjected to heat retrieval treatment to retrieve the antigens by using reveal solution (provided by Biocare Medical). This was performed in Decloaking Chamber (also provided by Biocare Medical) for 4 minutes. Reveal solution, as indicated by the producing company, is a revolutionary solution so that endogenous blocking of hydrogen peroxidase activity and protein block are omitted. After gradual cooling to room temperature, the sections were washed by PBS (phosphate buffered saline, $\mathrm{pH}$ 7.4).

With the exception of the negative control sections, all sections were covered with $200 \mu \mathrm{l}$ of the diluted primary antibody using revival series as the diluent. Two different monoclonal antibodies were used. The first antibody is monoclonal mouse Anti-Aspergillus (Clone Mab-WF-AF-1, CODE NO. M3564), and the second antibody is monoclonal mouse Anti-p53 (Do-1, provided by Santa Cruz Biotechnology) and incubated for 60 minutes at room temperature and then washed with PBS.

The sections including the negative controls were incubated for 10 minutes at room temperature with 200 $\mu \mathrm{l}$ of the diluted biotinylated secondary antibody followed by washing with PBS and incubation at room temperature with $200 \mu \mathrm{l}$ of the horseradish peroxidase-streptavidin for 10 minutes, then PBS washing. Chromogen (DAB) was added to visualize the reaction. DAB was applied for 2 - 5 minutes or until the reaction is developed.

The sections were immersed in tab water for 5 minutes, and counter-stained with Harris hematoxylin for 30 seconds to prevent interference with chromogen in case of having nuclear stain as p53. The sections were washed by tab water and dehydrated through ascending concentrations of alcohol, cleared, and mounted by DPX (Distrene Plasticizer Xylene).

Lastly, the sections were examined using light microscopy.

\subsection{Mach-4 Method}

This method has the same steps as LSAB Method except instead of the addition of the biotinylated secondary antibody and HRP-Streptavidin; Mach-4 reagent was incubated on the slides at room temperature for 10 minutes followed by washing with PBS.

\section{Results and Discussion}

In the current study, the detection of p53 and Aspergillus antigen in lung tissues were investigated using immunohistochemistry technology. The results show that the Aspergillus is present in all used sample $(100 \%$ of the infection) in the form of spores or hyphae (Table 1, Figures 1-3). It is also present within the blood vessels and within the interstitial spaces (Figure 2 and Figure 3). This high percent may be due to the highly specific and sensitive technique employed in this study (immunohistochemistry). Additionally, all cases in this study were from autopsy specimens, Autopsy specimens are more exposed to Aspergillus than other specimens. There may be relatively long time from death to obtain the specimens which may give a sufficient incubation time for the multiplication of Aspergillus.

When Mach-4 was used as a detection system which is a very highly sensitive detection system, the localization of Aspergillus is clearer even diffused within fluids (Figure 2 and Figure 3). The results show that all 
Table 1. The detection of Aspergillus spores/hyphae and p53 in 45 samples (lung tissues).

\begin{tabular}{|c|c|c|c|c|}
\hline \multirow{2}{*}{ Case \# } & \multicolumn{2}{|c|}{ Patient Data } & \multicolumn{2}{|c|}{ Aspergillus/p53 Occurrence } \\
\hline & Sex & Age & Apergillus & p53 \\
\hline 1 & M & 30 & +ve & +ve \\
\hline 2 & M & 1 & +ve & +ve \\
\hline 3 & M & 50 & +ve & +ve \\
\hline 4 & M & 33 & +ve & +ve \\
\hline 5 & M & 50 & +ve & +ve \\
\hline 6 & $\mathrm{~F}$ & 20 & +ve & +ve \\
\hline 7 & M & 57 & +ve & +ve \\
\hline 8 & M & 50 & +ve & $+v e$ \\
\hline 9 & M & 30 & +ve & +ve \\
\hline 10 & M & 60 & +ve & +ve \\
\hline 11 & M & 30 & +ve & +ve \\
\hline 12 & M & 28 & +ve & +ve \\
\hline 13 & $\mathrm{~F}$ & 30 & +ve & +ve \\
\hline 14 & $\mathrm{~F}$ & 39 & +ve & +ve \\
\hline 15 & M & 9 & +ve & +ve \\
\hline 16 & M & 10 days & +ve & +ve \\
\hline 17 & M & 5 months & +ve & +ve \\
\hline 18 & $\mathrm{~F}$ & 50 & +ve & +ve \\
\hline 19 & M & 40 & +ve & +ve \\
\hline 20 & M & 40 & +ve & +ve \\
\hline 21 & M & 86 & +ve & +ve \\
\hline 22 & $\mathrm{~F}$ & 19 & +ve & +ve \\
\hline 23 & M & 68 & +ve & +ve \\
\hline 24 & M & 60 & +ve & +ve \\
\hline 25 & M & 30 & +ve & +ve \\
\hline 26 & M & 20 & +ve & +ve \\
\hline 27 & M & 50 & +ve & +ve \\
\hline 28 & $\mathrm{~F}$ & 30 & +ve & +ve \\
\hline 29 & $\mathrm{~F}$ & 9 months & +ve & +ve \\
\hline 30 & $\mathrm{~F}$ & 31 & +ve & +ve \\
\hline 31 & M & 60 & +ve & +ve \\
\hline 32 & M & 29 & +ve & +ve \\
\hline 33 & M & 6 & +ve & +ve \\
\hline 34 & M & 30 & +ve & +ve \\
\hline 35 & $\mathrm{~F}$ & 37 & +ve & +ve \\
\hline 36 & $\mathrm{~F}$ & 2 & +ve & +ve \\
\hline 37 & $\mathrm{~F}$ & 40 & +ve & +ve \\
\hline 38 & M & 30 & +ve & +ve \\
\hline 39 & $\mathrm{~F}$ & 50 & +ve & +ve \\
\hline 40 & M & 40 & +ve & +ve \\
\hline 41 & M & 11 & +ve & +ve \\
\hline 42 & M & 6 & +ve & +ve \\
\hline 43 & M & 24 & +ve & +ve \\
\hline 44 & M & 50 & +ve & +ve \\
\hline 45 & M & 30 & $+\mathrm{ve}$ & $+\mathrm{ve}$ \\
\hline
\end{tabular}




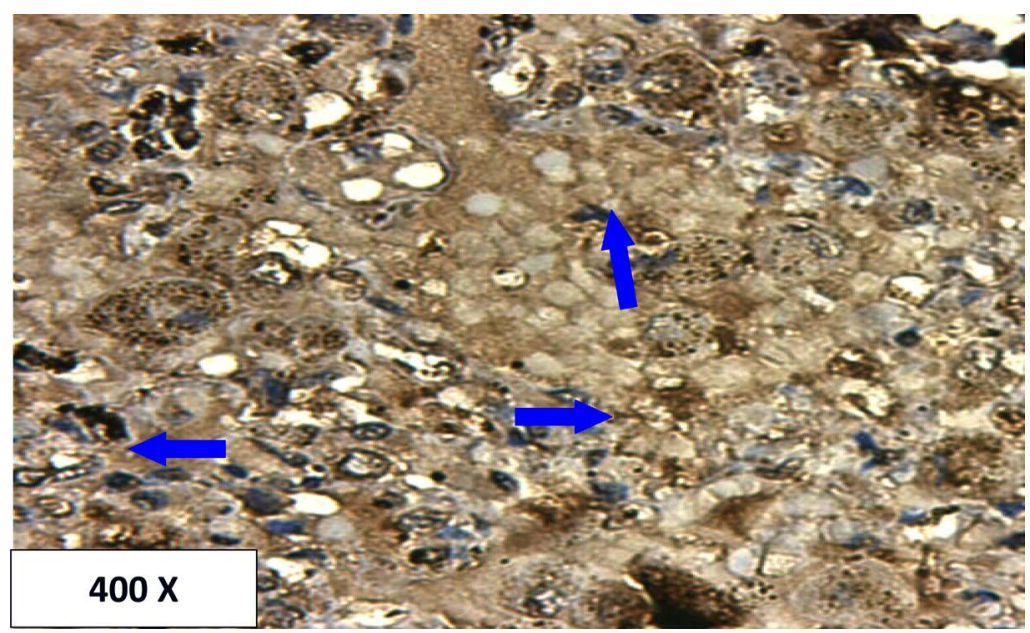

Figure 1. Localization of Aspergillus spores in lung tissue using LSAB-Method. Brown color at the tip of the arrows indicates positive.

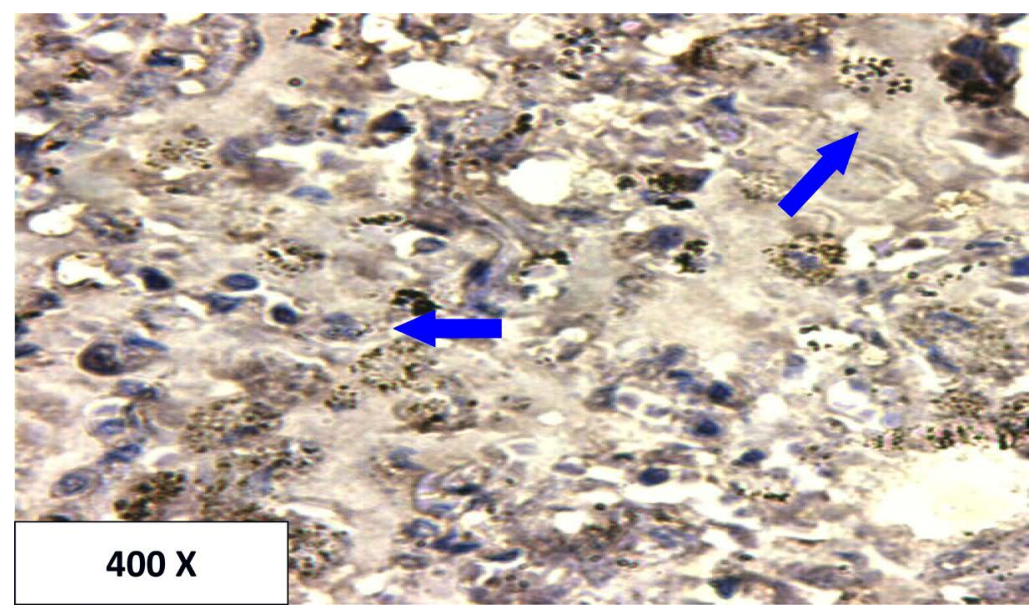

Figure 2. Localization of Aspergillus spores in lung tissue using Mach-4 method. Brown color at the tip of the arrows indicates positive.

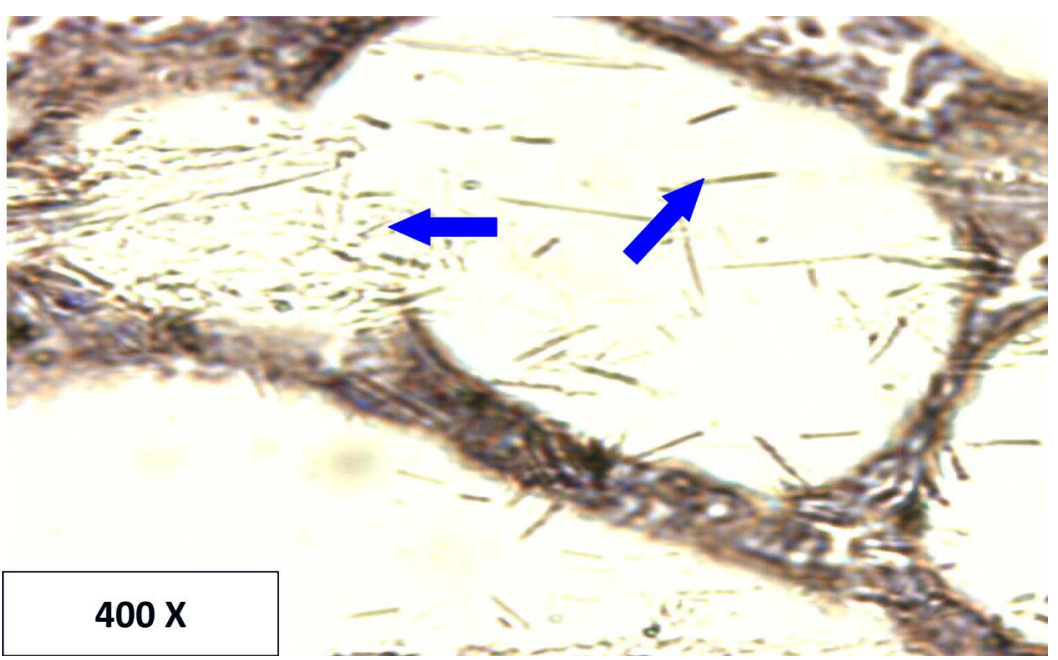

Figure 3. Localization of Aspergillus hyphae in lung tissue using Mach-4 method. Brown color at the tip of the arrows indicates positive. 


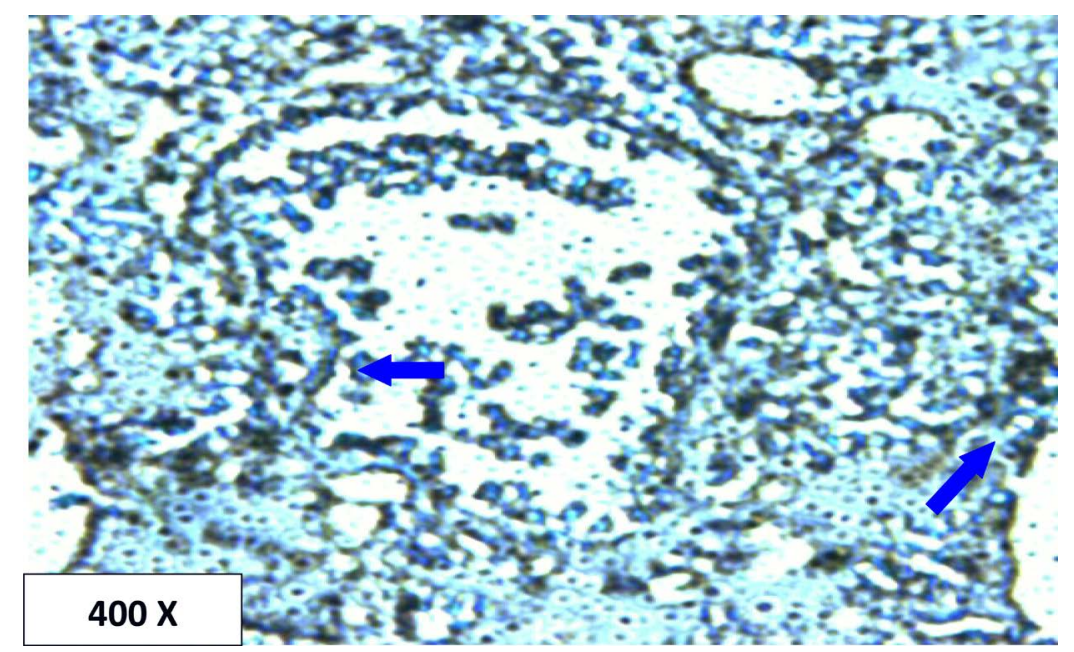

Figure 4. Immunostaining of p53 in nuclei of lung tissues using LSAB-Method. Brown color at the tip of the arrows indicates positive.

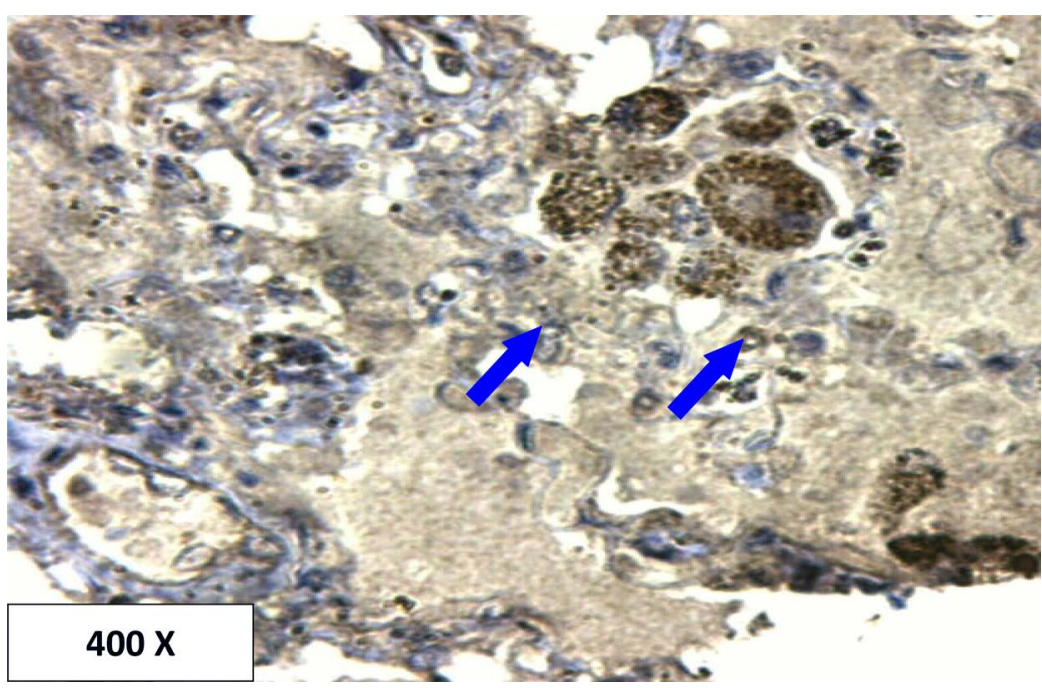

Figure 5. Immunostaining of p53 in lung tissues using Mach-4 method. Brown color at the tip of the arrows indicates positive.

infected samples have mutant p53 molecules (p53 was located in Aspergillus spores and hyphae) (Table 1, Figure 4 and Figure 5). p53 was present within nuclei of different cells within the lungs among which are the macrophages and blood vessels walls (Figure 4).

The results show that other variables such as age, sex, and lung pathology have no effect on the results (no change on the samples positivity for Aspergillus infection or presence of p53 in its spores and hyphae).

All used samples were positive for both Aspergillus infection and p53 in this study therefore we suggest there is a correlated relationship between these two variables.

According to this study, it is safe to posit that the mutant p53 molecules may be used by Aspergillus for its multiplication. Seemingly it is a biological behavior of Aspergillus to produce p53. The fate of the p53 is questioned, is it going to interact with the human cells initiating cancer? Further experimental investigations are required to determine such pathway.

\section{Conclusion}

In conclusion, this study shows that Aspergillus is a producing agent for p53 and Aspergillus pathogenicity is caused by production of p53. 


\section{Acknowledgements}

The authors would like to thank Dr. Ali Shoter for allowing to use his lab space and equipments at the center of forensic medicine-Irbid, Jordan. Heartfelt gratitude is extended to Miss Amal Zahran and Mr Ali Otoom for their guidance and help as well as Dr. Mohammed Abu Saleh and Dr Ahmed Abu Taleb for their assistance in statistical analysis.

\section{References}

[1] Schumacher, B., Hofmann, K., Boulton, S. and Gartner A. (2001) The C. elegans Homolog of the p53 Tumor Suppressor Is Required for DNA Damage-Induced Apoptosis. Current Biology, 11, 1722-1727.

http://www.ncbi.nlm.nih.gov/pubmed/11696333

http://dx.doi.org/10.1016/S0960-9822(01)00534-6

[2] Michael, O., Young, L., DiComo, C., Karim, F., Belvin, M., Robertson, S., Whittaker, K., Demsky, M., Fisher, W., Buchman, A., Duyk, G., Friedman, L., CPrives, C. and Kopczynski, C. (2000) Drosophila p53 Is a Structural and Functional Homolog of the Tumor Suppressor p53. Cell, 101, 91-101.

http://www.sciencedirect.com/science/article/pii/S0092867400806261 http://dx.doi.org/10.1016/S0092-8674(00)80626-1

[3] Young, R.C., Bennett, J.E., Vogel, C.L., Carbone, P.P. and DeVita, V.T. (1970) Aspergillosis: The Spectrum of Disease in 98 Patients. Medicine, 49, 147-73. http://www.ncbi.nlm.nih.gov/pubmed/4913991 http://dx.doi.org/10.1097/00005792-197003000-00002

[4] Van Burik, J.A. and Magee, P.T. (2001) Aspects of Fungal Pathogenesis in Humans. Annual Review of Microbiology, 55, 743-772. http://www.ncbi.nlm.nih.gov/pubmed/11544373 http://dx.doi.org/10.1146/annurev.micro.55.1.743

[5] Marterre, W.F., Mong, A.T. and Pulito, A.R. (1992) Locally Invasive Aspergillosis of the Bowel. Journal of Pediatric Surgery, 27, 1611-1613. http://www.ncbi.nlm.nih.gov/pubmed/1469594 http://dx.doi.org/10.1016/0022-3468(92)90527-e

[6] Mori, T., Matsumara, M., Ymada, K., Irie, S., Oshimi, K., Suda, K., Oguri, T. and Ichinoe, M. (1998) Systemic Aspergillosis Caused by an Aflatoxin-Producing Strain of Aspergillus flavus. Medical Mycology, 36, 107-112. http://informahealthcare.com/doi/abs/10.1080/02681219880000171 http://dx.doi.org/10.1080/02681219880000171

[7] Smela, M.E., Currier, S.S., Bailey, E.A. and Essigmann, J.M. (2001) The Chemistry and Biology of Aflatoxin B(1): from Mutational Spectrometry to Carcinogenesis. Carcinogenesis, 22, 535-545.

http://www.ncbi.nlm.nih.gov/pubmed/11285186

http://dx.doi.org/10.1093/carcin/22.4.535

[8] Livingstone, L.R., White, A., Sprouse, J., et al. (1992) Altered Cell Cycle Arrest and Gene Amplification Potential Accompany Loss of Wild-Type p53. Cell, 70, 923-935. http://www.ncbi.nlm.nih.gov/pubmed/1356076 http://dx.doi.org/10.1016/0092-8674(92)90243-6

[9] Hussain, S.P. and Harris, C.C. (2000) Molecular Epidemiology and Carcinogenesis: Endogenous and Exogenous Carcinogens. Mutation Research, 462, 311-322. http://www.ncbi.nlm.nih.gov/pubmed/10767641 http://dx.doi.org/10.1016/s1383-5742(00)00015-6

[10] Huncharek, M., Kupelnick, B., Geschwind, J.F., et al. (2000) Prognostic Significance of p53 Mutations in Non-Small Cell Lung Cancer: A Meta-Analysis of 829 Cases from Eight Published Studies. Cancer Letter, 153, 219-226. http://www.ncbi.nlm.nih.gov/pubmed/10779652 http://dx.doi.org/10.1016/S0304-3835(00)00381-5 